\title{
Inhibited autonomy for promoting physical health: qualitative analysis of narratives from persons living with severe mental illness
}

Miharu Nakanishi, Shintaro Tanaka, George Kurokawa, Shuntaro Ando, Syudo Yamasaki, Masato Fukuda, Kiyohisa Takahashi, Takuya Kojima and Atsushi Nishida

\section{Background}

Autonomy is a key factor in the reduction of inequitable physical healthcare among people with severe mental illness compared with the general population.

\section{Aims}

To clarify the critical mechanism underlying autonomy in physical health promotion based on the perspectives of people with severe mental illness.

\section{Method}

We employed a conventional content analysis of narrative data from the Healthy Active Lives in Japan (HeAL Japan) workshop meetings.

\section{Results}

'Inhibited autonomy' was extracted as a central component and shaped by the users' experiences, both in a healthcare setting and in real life. This component emerged based on the lack of an empowerment mechanism in psychiatric services.

\section{Conclusions}

A barrier to the encouragement of autonomy in physical health promotion was found in current psychiatric services. An effective strategy should be explored to foster an empowerment mechanism in psychiatric and mental health services.

\section{Declaration of interest}

None.

\section{Keywords}

Healthy lifestyle; health promotion; mental health service: personal autonomy; schizophrenia.

\section{Copyright and usage}

(c) The Royal College of Psychiatrists 2019. This is an Open Access article, distributed under the terms of the Creative Commons Attribution-NonCommercial-NoDerivatives licence (http://creativecommons.org/licenses/by-nc-nd/4.0/), which permits noncommercial re-use, distribution, and reproduction in any medium, provided the original work is unaltered and is properly cited. The written permission of Cambridge University Press must be obtained for commercial re-use or in order to create a derivative work.
Inequality in physical health has been raised as a concern among people with severe mental illness compared with the general population. Schizophrenia and related disorders are associated with increased mortality and reduced life expectancy. ${ }^{1,2}$ The majority of deaths are due to poor physical health and related cardiovascular disease, ${ }^{1,3}$ which are partially attributable to antipsychotic medication, ${ }^{4-6}$ psychotic symptoms, ${ }^{6,7}$ disparities in access to physical healthcare among people with severe mental illness ${ }^{6,8}$ and suboptimal lifestyle behaviour including smoking, an unhealthy diet and lack of exercise. ${ }^{6,9}$ Therefore, treatment actions are required to address suboptimal lifestyle choices and to monitor and treat crucial physical parameters. ${ }^{10}$

Several physical health promotion programmes have been developed to improve lifestyle behaviours among people with severe mental illness. Although some intervention trials were successful in increasing exercise ${ }^{11}$ and reducing weight, ${ }^{12,13}$ the effect of these interventions is inconsistent ${ }^{14}$ and is suboptimal on anthropometric measures. ${ }^{15}$ Furthermore, treatment drop-out is high, particularly for physical activity interventions among people with schizophrenia. ${ }^{16}$ Autonomy can play a key role in the continuation of physical activity programs ${ }^{17}$ and healthy lifestyle behaviours. ${ }^{18,19}$ However, little is known regarding the critical mechanism of autonomy in relation to physical health promotion. Elucidating this mechanism based on the perspectives of people with severe mental illness could reveal an effective strategy to enhance autonomous motivation for physical health promotion.

\section{Method}

\section{Design}

We adopted a qualitative study design.

\section{Setting}

Data collection was conducted in Japan from October 2016 to August 2017. In Japan, the mental healthcare system has predominantly remained dependent on hospital-based services, and community mental health services have not functioned well compared to other countries within the Organisation for Economic Co-operation and Development. ${ }^{20}$ Excessive mortality among individuals with severe mental illness was observed in Japan as well as in other countries. $^{2}$

\section{Procedure}

We collected narrative data from two different sources, both of which were conducted in the Healthy Active Lives in Japan (HeAL Japan) workshop meeting: (a) a panel discussion among service users and (b) a group discussion involving service users, carers, service providers and other stakeholders.

HeAL Japan began in 2014 with support from the international HeAL group (http://www.iphys.org.au/what-is-heal). HeAL Japan aims to promote better physical health among people with severe mental illness in Japan. During 2015-16, we held annual workshops 
for networking individuals who are committed to physical health promotion in people with mental illness. A total of 40-60 participants attended each workshop and they were divided into 5-8 groups. Each group consisted of eight to ten members of service users, carers, service providers and other stakeholders. Participants were recruited via an online announcement and opportunistic sampling of the HeAL Japan board members.

\section{Ethical considerations}

Participation in the workshop was voluntary. Written informed consent was obtained from all participants, and participant anonymity has been preserved. The study conforms to the provisions of the Declaration of Helsinki (as revised in 2013). The study was approved by the ethical committee of the Tokyo Metropolitan Institute of Medical Science (number 16-37).

\section{Data collection}

\section{Panel discussion}

Three service users were invited to participate in a workshop on physical health among people with mental illness (HeAL Japan symposium) as panellists. The panel discussion was held in October 2016. A session facilitator (G.K.) was a mental health service user and a peer supporter. He has been involved in the activities of the HeAL Japan initiatives (http://healjapan.info/) as one of the co-representatives. Participants of the panel discussion were selected by recommendations from the HeAL Japan board members. Recommendations were made according to the following criteria: service users (a) who were not experiencing acute deterioration of mental state, (b) who were able to report their own experiences to unfamiliar persons and (c) who were willing to discuss physical health in the context of living with mental illness. Before the panel discussion, a group discussion was held to clarify issues regarding physical health among people with mental illness from the perspectives of service users. The panel discussion consisted of short presentations from these service users and discussions among them and the facilitator. We made arrangements in advance of the meeting and acknowledged withdrawal due to deterioration of psychological conditions. We video recorded this session and transcribed the narratives of the service users to text.

\section{Group discussion}

Group discussions among service users, carers and service providers were held at the HeAL Japan workshop in October 2016 after the panel discussion by service users. We classified the participants according to the results of a self-recorded questionnaire as follows: psychiatrist, nutritionist, service user, user's family, psychiatric service provider, science researcher, volunteer supporter and a member of the media. We balanced each group of participants in terms of social class and gender, and achieved a mixed composition using the classification mentioned above.

The members of each group discussed the factors that promote and prohibit improved physical health among people with mental illness and the minutes were summarised. We used these recorded minutes as the data in this study.

\section{Study participants}

\section{Service users in the panel discussion}

Three service users participated in the panel discussion as panellists. They used a fictitious name, were males aged $30-50$ years and had been diagnosed with schizophrenia. The session facilitator was a male aged around 40 years with depressive disorder.

\section{Participants in the group discussion}

There was a total of 37 participants in the group discussion. Participants included healthcare service providers, carers, service users, students and people involved in the promotion of physical health among those with mental illness in the community.

\section{Data analysis}

Qualitative material from the narratives of service users in the panel discussion and the minutes from the group discussions among service users, carers and service providers were analysed using conventional content analysis. ${ }^{21}$ The categories and subcategories were discussed and reviewed by the authors, including service users and researchers. The finalised model of categories and subcategories was approved by all service users who participated in the panel discussion.

\section{Results}

Although contributing factors for physical health promotion were sought in the panel discussion and group discussions, the participants' narratives mainly comprised barriers to the encouragement of autonomy in physical health promotion among people with severe mental illness. The content analysis consequently extracted the components of these barriers (Fig. 1).

'Inhibited autonomy' was extracted as a central component of the barriers to physical health promotion. Other components included 'healthcare setting', 'real life' and 'lack of an empowerment mechanism'.

\section{Inhibited autonomy}

The inhibited autonomy component was shaped by the service users' experiences both in the healthcare setting and in real life. The healthcare setting component comprised barriers to physical health promotion at routine psychiatric services. The real life component comprised barriers to physical health promotion in daily living due to side effects of antipsychotic medication and symptoms of mental illness. The inhibited autonomy component itself comprised 'difficulties in maintaining attention on one's own physical health promotion' and 'internalised feeling of how the person is perceived in a mental healthcare setting'.

\section{Difficulties in maintaining attention on one's own physical health promotion}

Although participants agreed that poor physical health (such as reduced life expectancy and elevated risk of diabetes) was of concern for people with severe mental illness, they felt it was difficult to promote their own physical health. For example, one of the participants of the panel discussion stated that he had lost interest in promoting his own physical health due to severe fatigue as a result of the symptoms of schizophrenia.

\section{Internalised feeling of how the person is perceived in a mental healthcare setting}

The challenges in physical health promotion could be attributed to the small selection of options that were offered by psychiatric and mental health services. For example, one of the participants of the panel discussion described his experiences with psychiatrists as being treated as a research participant for a new drug against his will. He experienced fear of psychiatrists because he recognised his own mental disease condition as stable and had no desire to change his medication. 


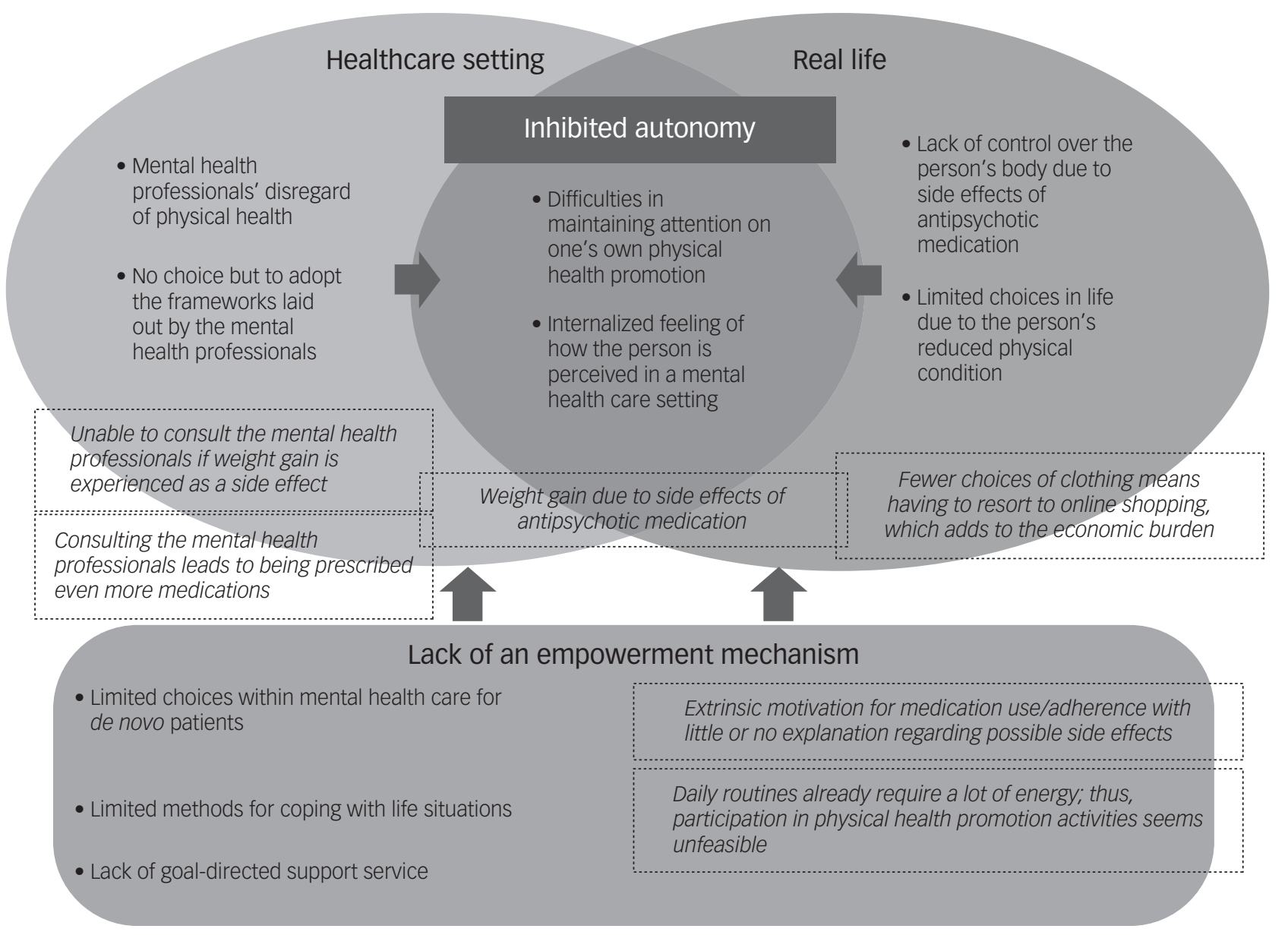

Fig. 1 The mechanism underlying inhibited autonomy in physical health promotion among persons with severe mental illness. The two circles present the challenges experienced by the person with severe mental illness in a healthcare setting and in real life. The overlapping area presents inhibited autonomy that was shaped by their experiences in a healthcare setting and in real life. The square at the bottom presents the lack of empowerment mechanism that was the basis of the emerging inhibited autonomy. The comments in the dotted squares are examples of each component and were extracted from narrative data.

Other participants also described their experiences with psychiatric services as offering limited options. These experiences in mental healthcare settings induced the internalised feeling of low self-determination and inhibited their autonomy in physical health promotion.

\section{Healthcare setting}

The healthcare setting component shaped inhibited autonomy, as did experiences in real life. This component referred to the situation wherein the participants had little sense of self-determination in routine psychiatric services. The component consisted of 'mental health professionals' disregard of physical health' and 'no choice but to adopt the frameworks laid out by the mental health professionals'.

\section{Mental health professionals' disregard of physical health}

The participating service users stated that there were fewer persons available to provide advice for physical health issues compared with that for mental health issues. They also described their feeling that mental health professionals disregarded physical health issues such as side effects of antipsychotic medication:

Facilitator: I see. Medications in general include side effects, but were you all provided with any explanation from your doctor regarding the possible risks at the time of initial prescription?

Participant A: I was repeatedly instructed to 'just take them for now'. I was being hospitalised and was told 'Your illness will worsen if you don't take the medications even just for 1 day'. He insisted that 'I just have to take the medications'.

Participant B: I used to consult the doctors but, if I did, they would prescribe more medications. For about 10 years, I was on many types of medication but I have few memories of those days and all are disorganised. These days I... not all side effects are the same. I do consult if it's severe but if not, I just put up with it.

Participant B: I have changed hospitals about three times now. Two of them were small, private clinics. The current one is quite big. The doctors change about once a year, and new doctors sometimes try prescribing new medications. If, for example, my condition isn't all that well, then I might be willing to take their advice. But if my condition is quite stable, then that would just be a daunting experience for me.

The participants reported that psychiatrists had prescribed new antipsychotic medication without adequate explanation of the potential side effects. Moreover, participants tended to hesitate 
when consulting psychiatrists with regard to medication for two reasons. First, they worried that the psychiatrist would deem the inquiry regarding medication as a confession of their dissatisfaction with the treatment. Second, they were concerned that the psychiatrist would assume the inquiry as a sign of mental health deterioration and increase the antipsychotic medication in response to the inquiry.

No choice but to adopt the frameworks laid out by the mental health professionals

Both service users and service providers expressed their interest in using complementary and alternative therapies for mental illness such as exercise, nutrition management and meditation. However, participants of the panel discussion mentioned that they have faced a binary decision between acceptance of everything in the prescription or no treatment given in psychiatric and mental health services:

Participant B: Now that I have taken this much antipsychotic medication, I just have to keep taking it. However, sometimes I wish, if there was another me, I could have sought ways to cope with the problem without taking any medication.

\section{Real life}

The real life component shaped the inhibited autonomy component as did experiences in healthcare setting. This component referred to the situation wherein the participants encountered difficulties in daily living due to the side effects of antipsychotic medication and symptoms of mental illness. The component consisted of 'lack of control over the person's body due to side effects of antipsychotic medication' and 'limited choices in life due to the person's reduced physical condition'.

\section{Lack of control over the person's body due to the side effects of antipsychotic medication}

Some service users reported that their difficulties in daily life had been caused by the side effects of antipsychotic medication. They expressed the feeling of a lack of control over their body:

Facilitator: When you hear the term 'physical health', what do you think of in relation to your physical state? So, you are experiencing mental illness, but is there anything else, including feelings of exhaustion, that you can think of?

Participant C: Ah, about my physical health. My hands twitch, my eyes are being pulled up, and I can't look down. I also feel like pulling out my tooth.

Participant A: For me, it's about the side effects. I'm on a medication called olanzapine, and I've put on about $25 \mathrm{~kg}$ in weight. I think that if I can shed a bit more weight, I might feel lighter or more comfortable.

Participant B: For example, I have muscle spasms all over my body. Just occasionally, that wakes me up. I also started putting on weight since I started taking medication. I've been taking it for about 20 years, and it has been a struggle losing weight.

Following the exchange of reflections at the panel discussion, the session facilitator asked about physical health promotion. However, the participants responded by talking about side effects of antipsychotic medication. This conversation may have reflected their perception of side effects by antipsychotic medications as a dominant force in their lives.
Limited choices in life due to the person's reduced physical condition

One narrative from a participant illustrated the issue of obesity that had appeared over the 20 years that the person had been living with schizophrenia. After he was diagnosed with the disease, he had to avoid entering a clothes shop because he could not cope with the noisy atmosphere. When antipsychotic medication was prescribed, he did not receive sufficient explanation of the side effects of antipsychotic medication. He then noticed that his weight gain may have been influenced by the antipsychotic medication; nonetheless, he experienced difficulty in asking the psychiatrist about the weight gain because he assumed that asking the question was a violation of the 'authority of a medical doctor'.

Other participants demonstrated the same tendency to avoid consulting psychiatrists on side effects. They commented that psychiatrists could deem the service user's request for consultation on medication to be a negative attitude toward the prescription, or could add other prescriptions of psychiatric medication to alleviate the side effects.

\section{Lack of an empowerment mechanism}

The lack of an empowerment mechanism component was based on the situation wherein inhibited autonomy was shaped by the service users' experiences both in a healthcare setting and in real life. This component consisted of 'limited choices within mental healthcare for de novo patients', 'limited methods for coping with life situations' and 'lack of goal-directed support service'.

\section{Limited choices within mental healthcare for de novo patients}

As described in the real life component, the participants experiencing their first episode of psychosis were prescribed antipsychotic medication with little explanation of possible side effects. The initial encounter with psychiatric services was followed by extrinsic motivation to take antipsychotic medication and use mental health services.

\section{Limited methods for coping with life situations}

Some service users reported suboptimal lifestyle behaviours. They assumed that the unhealthy lifestyle behaviours might have arisen to cope with daily living situations due to limited choices:

Facilitator: And you started smoking. Was this anything to do with the stress?

Participant C: It was.

Facilitator: What was it like?

Participant C: At times, I felt hopeless.

Facilitator: How did smoking cigarettes make you feel?

Participant C: It temporarily contracts the blood vessels, so it did make me feel revitalised.

Participant A: For me, it's about the weight gain, the obesity. When it comes to how much I eat. I guess I would be able to shed weight if I reduced my calorie intake. But then again, whenever I feel stressed or burdened, I can't help eating more during mealtimes. I also snack more on things like potato chips and chocolates. I think that the mental instability causes binge eating.

Participants of the panel discussion assumed few stress coping mechanisms aside from alcohol, junk food, binge eating and smoking. They also had limited opportunities to explore other stress coping mechanisms and described it unfeasible to simply stop suboptimal lifestyle behaviours without any alternative methods. 
Lack of goal-directed support service

As outlined previously, the participants felt that they had little selfdetermination in terms of their treatment. The psychiatric service providers focused more on symptom management with regard to mental illness rather than on empowerment of service users. At the group discussion, a psychiatrist stated that paternalism of healthcare professionals still exists in psychiatry, and that mental health professionals tend to decide on a treatment plan in disregard of the person's value.

\section{Discussion}

This study performed a qualitative analysis of narrative data from service users to clarify the critical mechanism of autonomy regarding physical health promotion. Inhibited autonomy was extracted as a central component involved in the lack of empowerment of people receiving psychiatric services in Japan.

Our study first elucidated the impact of the decision-making process in psychiatric and mental health services on autonomy regarding physical health among persons with severe mental illness. In Japan, psychosocial approaches for the treatment of psychosis remain under development. ${ }^{20}$ Thus, antipsychotic medication is the only intervention available for mental health professionals to treat persons with severe mental illness. Additionally, compulsory admission is still common in Japanese psychiatric hospitals (12 9114 of 284806 in-patients [45.3\%] on 30 June 2015). ${ }^{22}$ Decision-making in Japanese mental health settings thus occurs in a clinician-led manner, which shapes an inhibited sense of control and empowerment in the patient. This may be a barrier to the encouragement of autonomy in physical health promotion. An effective strategy to improve the care provided to persons with mental illness should be explored, which should include advocacy and peer support as well as referral to flexible physical healthcare services.

The participating service users experienced difficulties in consulting psychiatrists on the side effects of antipsychotic medications. This is in agreement with a previous study that suggested difficulties in reporting side effects to the psychiatrist among service users in Japan. ${ }^{23}$ With regard to the experience of healthcare providers, the diagnosis of schizophrenia negatively affects their expectation of the patients' competence to manage their own health and personal affairs. ${ }^{24}$ In turn, the diagnosis of schizophrenia is associated with fewer referrals to a weight-reduction programme. $^{24}$ Psychiatrists often omitted the physical health assessment of patients who received antipsychotic medication. ${ }^{25}$ The disregard of physical health may have led to inequity in access to physical healthcare and inhibited autonomy regarding physical health promotion among persons with severe mental illness.

This study had several limitations. Firstly, as the narrative data was collected from the group discussion and panel discussion at the workshop, our data may have lacked the narrative from those persons with more severe mental conditions who could not attend the workshop. The nature of data collection in our study did not allow a further in-depth reflection on the issue of autonomy. However, our content analysis enabled us to determine the mechanism underlying the lack of patient empowerment in mental health settings. Secondly, panel discussion participants were only male, and the majority of the audience at the panel discussion were psychiatric service providers. These biases could lead to limited generalisability of this study.

Future research should explore an effective strategy to foster an empowerment mechanism in the strategies provided by psychiatric and mental health services.
Miharu Nakanishi, RN, PhD, Chief Researcher, Mental Health and Nursing Research Team, Mental Health Promotion Project, Tokyo Metropolitan Institute of Medical Science, Japan; Shintaro Tanaka, MA, Research Assistant, Mental Health Promotion Project, Tokyo Metropolitan Institute of Medical Science, Japan; George Kurokawa, Peer Staff, Social Welfare Corporation Sudachikai, Japan; Shuntaro Ando, MD, PhD, Lecturer, Department of Neuropsychiatry, Graduate School of Medicine, The University of Tokyo, Japan; Syudo Yamasaki, PhD, Chief Researcher, Mental Health Promotion Project, Tokyo Metropolitan Institute of Medical Science, Japan; Masato Fukuda, MD, PhD, Professor, Department of Psychiatry and Neuroscience, Gunma University Graduate School of Medicine, Japan; Kiyohisa Takahashi, MD, PhD, Director, Mental Health and Neurology Center, Japan; Takuya Kojima, MD, PhD, Vice-Director, Ohmiya Kousei Hospital, Japan; Atsushi Nishida, PSW, PhD, Project Leader, Mental Health Promotion Project, Tokyo Metropolitan Institute of Medical Science, Japan.

Correspondence: Miharu Nakanishi, Mental Health Promotion Project, Tokyo Metropolitan Institute of Medical Science, 2-1-6 Kamikitazawa, Setagaya-ku, Tokyo 156-8506, Japan. Email: mnakanishi-tky@umin.ac.jp

First received 27 Jul 2018, final revision 8 Nov 2018, accepted 11 Nov 2018

\section{Funding}

This work was supported by the Japan Agency for Medical Research and Development (16dk0307059) and the Japan Society for the Promotion of Science KAKENHI via a Grant-inAid for Challenging Exploratory Research under grant number JP16K13499. The article represents the personal views of the authors and is not the official view of the following organisations: Tokyo Metropolitan Institute of Medical Science, Social Welfare Corporation Sudachikai, the University of Tokyo, Gunma University Graduate School of Medicine, Japan Foundation for Neuroscience and Mental Health or Ohmiya Kousei Hospital.

\section{References}

1 Lawrence D, Hancock K, Kiseky S. The gap in life expectancy from preventable physical illness in psychiatric patients in Western Australia: retrospective analysis of population based registers. BMJ 2013; 346: f2539.

2 Kondo S, Kumakura Y, Kanehara A, Nagato D, Ueda T, Matsuoka T, et al. Premature deaths among individuals with severe mental illness after discharge from long-term hospitalisation in Japan: a naturalistic observation during a 24year period. BJPsych Open 2017; 3: 193-5.

3 Correll CU, Solmi M, Veronese N, Bortolato B, Rosson S, Santonastaso P, et al. Prevalence, incidence and mortality from cardiovascular disease in patients with pooled and specific severe mental illness: a large-scale meta-analysis of 3211768 patients and 113383368 controls. World Psychiatry 2017; 16: 163-80.

4 Correll CU, Detraux J, De Lepeleire J, De Heart M. Effects of antipsychotics, antidepressants and mood stabilizers on risk for physical diseases in people with schizophrenia, depression and bipolar disorder. World Psychiatry 2015; 14: 119-36.

5 Meyer JM, Davis VG, Goff DC, McEvoy JP, Nasrallah HA, Davis SM, et al. Change in metabolic syndrome parameters with antipsychotic treatment in the CATIE Schizophrenia Trial: prospective data from phase 1. Schizophr Res 2008; 101: 273-86.

6 Hert MD, Correll CU, Bobes J, Cetkovich-Bakmas M, Cohen D, Asai I, et al. Physical illness in patients with severe mental disorders. I. Prevalence, impact of medications and disparities in health care. World Psychiatry 2011; 10: 52-77.

7 Moreno C, Nuevo R, Chatterji S, Verdes E, Arango C, Ayuso-Mateos JL. Psychotic symptoms are associated with physical health problems independently of a mental disorder diagnosis: results from the WHO World Health Survey. World Psychiatry 2013; 12: 251-7.

8 Moore S, Shiers D, Daly B, Mitchell AJ, Gaughran F. Promoting physical health for people with schizophrenia by reducing disparities in medical and dental care. Acta Psychiatr Scand 2015; 132: 109-21.

9 Scott D, Happel B. The high prevalence of poor physical health and unhealthy lifestyle behaviours in individuals with severe mental illness. Issues Ment Health Nurs 2011; 32: 589-97.

10 De Hert M, Cohen D, Bobes J, Cetkovich-Bakmas M, Leucht S, Ndetei DM, et al. Physical illness in patients with severe mental disorders. II. Barriers to care, monitoring and treatment guidelines plus recommendations at the system and individual level. World Psychiatry 2011; 10: 138-51.

11 Bartels SJ, Pratt SI, Aschbrenner KA, Barre LK, Jue K, Wolfe RS, et al. Clinically significant improved fitness and weight loss among overweight persons with serious mental illness. Psychiatr Serv 2013; 64: 729-36.

12 Wu RR, Zhao JP, Jin H, Shao P, Fang MS, Guo XF, et al. Lifestyle intervention and metformin for treatment of antipsychotic-induced weight gain. JAMA 2008; 29 185-93. 
13 Daumit GL, Dickerson FB, Wang NY, Dalcin A, Jerome GJ, Anderson CAM, et al. A behavioral weight-loss intervention in persons with serious mental illness. $N$ Engl J Med 2013; 368: 1594-602.

14 Firth J, Cotter J, Elliott R, French P, Yung AR. A systematic review and meta-analysis of exercise interventions in schizophrenia patients. Psychol Med 2015; 45 1343-61.

15 Rosenbaum S, Tiedemann A, Sherrington C, Curtis J, Ward PB. Physical activity interventions for people with mental illness: a systematic review and metaanalysis. J Clin Psychiatry 2014; 75: 964-74.

16 Vancampfort D, Rosenbaum S, Schuch FB, Ward PB, Probst M, Stubbs B. Prevalence and predictors of treatment dropout from physical activity interventions in schizophrenia: a meta-analysis. Gen Hosp Psychiatry 2016; 39 15-23.

17 Vancampfort D, De Hert $M$, Vansteenkiste $M$, De Herdt A, Scheewe TW Soundy $A$, et al. The importance of self-determined motivation towards physical activity in patients with schizophrenia. Psychiatry Res 2013; 210: 812-8.

18 Carney R, Cotter J, Bradshaw T, Yung AR. Examining the physical health and lifestyle of young people at ultra-high risk for psychosis: a qualitative study involving service users, parents and clinicians. Psychiatry Res 2017; 255: 87-93.

19 Vancampfort D, Vansteenkiste M, De Hert M, De Hert A, Soundy A, Stubbs B et al. Self-determination and stage of readiness to change physical activity behaviour in schizophrenia. Ment Health Phys Act 2014; 7: 171-6.
20 Organisation for Economic Co-operation and Development (OECD). OECD Reviews of Health Care Quality: Japan 2015: Raising Standards. OECD Publishing, 2015

21 Hsieh H-F, Shannon SE. Three approaches to qualitative content analysis. Qual Health Res 2005; 15: 1277-88.

22 National Center of Neurology and Psychiatry. The survey of Mental Health (630 survey). Department of Mental Health Policy and Evaluation, National Institute of Mental Health, National Center of Neurology and Psychiatry, 2017 (http:// www.ncnp.go.jp/nimh).

23 Hatano M, Kamei H, Kato A, Takeuchi I, Hanya M, Uno J, et al. Assessment of the latent adverse events of antipsychotic treatment using a subjective questionnaire in Japanese patients with schizophrenia. Clin Psychopharmacol Neurosci 2017; 15: 132-7.

24 Sullivan G, Mittal D, Reaves CM, Haynes TF, Han X, Mukherjee S, et al. Influence of schizophrenia diagnosis on providers' practice decisions. J Clin Psychiatry 2015; 76: 1068-74.

25 Laugharne J, Waterreus AJ, Castle DJ, Dragovic M. Screening for the metabolic syndrome in Australia: a national survey of psychiatrists' attitudes and reported practice in patients prescribed antipsychotic drugs. Australas Psychiatry 2015; 24: $62-6$. 